\title{
Single axon branching analysis in rat thalamocortical projection from the anteroventral thalamus to the granular retrosplenial cortex
}

\author{
Saori Odagiri ${ }^{1}$, Reiko Meguro' ${ }^{1}, Y_{0 s h i y a ~ A s a n o}{ }^{1}$, Toshiki Tani $^{2}$ and Noritaka Ichinohe ${ }^{1,2,3}$ * \\ 1 Department of Neuroanatomy, Histology and Cell Biology, Graduate School of Medicine, Hirosaki University, Hirosaki, Japan \\ ${ }^{2}$ Department of Anatomical Science, Graduate School of Medicine, Hirosaki University, Hirosaki, Japan \\ ${ }^{3}$ National Center of Neurology and Psychiatry, Department of Ultrastructural Research, National Institute of Neuroscience, Kodaira, Japan
}

\section{Edited by:}

Jose L. Lanciego, University of

Navarra, Spain

Reviewed by:

Francisco Clasca, Autonoma University, Spain

Yasushi Kobayashi, National Defense

Medical College, Japan

*Correspondence:

Noritaka Ichinohe, National Center of Neurology and Psychiatry,

Department of Ultrastructural

Research, National Institute of

Neuroscience, 4-1-1 Ogawa-Higashi,

Kodaira, Tokyo 187-8502, Japan.

e-mail: nichino@ncnp.go.jp

\begin{abstract}
The granular retrosplenial cortex (GRS) in the rat has a distinct microcolumn-type structure. The apical tufts of dendritic bundles at layer I, which are formed by layer II neurons, colocalize with patches of thalamic terminations from anteroventral (AV) thalamic nucleus. To further understand this microcolumn-type structure in the GRS, one of remaining questions is whether this structure extends into other layers, such as layers III/IV. Other than layer I, previous tracer injection study showed that AV thalamic nucleus also projects to layer III/IV in the GRS. In this study, we examined the morphology of branches in the GRS from the $\mathrm{AV}$ thalamus in single axon branch resolution in order to determine whether $\mathrm{AV}$ axon branches in layer III/IV are branches of axons with extensive branch in layer I, and, if so, whether the extent of these arborizations in layer III/IV vertically matches with that in layer I. For this purpose, we used a small volume injection of biotinylated dextran-amine into the AV thalamus and reconstructing labeled single axon branches in the GRS. We found that the AV axons consisted of heterogeneous branching types. Type 1 had extensive arborization occurring only in layer la. Type 2 had additional branches in III/IV. Types 1 and 2 had extensive ramifications in layer la, with lateral extensions within the previously reported extensions of tufts from single dendritic bundles (i.e., 30-200 $\mu \mathrm{m}$; mean $78 \mu \mathrm{m}$ ). In type 2 branches, axon arborizations in layer III/IV were just below to layer la ramifications, but much wider (148-533 $\mu \mathrm{m}$ : mean, $341 \mu \mathrm{m}$ ) than that in layer la axon branches and dendritic bundles, suggesting that layer-specific information transmission spacing existed even from the same single axons from the AV to the GRS. Thus, microcolumn-type structure in the upper layer of the GRS was not strictly continuous from layer I to layer IV. How each layer and its components interact each other in different spatial scale should be solved future.
\end{abstract}

Keywords: cortical modular organization, dendritic bundle, single axon reconstruction, cortical layer, limbic cortex

\section{INTRODUCTION}

The granular retrosplenial cortex (GRS) in rats is implicated in a wide range of behaviors, including visual and vestibular integration, path integration and spatial navigation, and certain aspects of learning and memory (Cooper and Mizumori, 2001; Garden et al., 2009; Vann et al., 2009; Aggleton, 2010). The retrosplenial cortex is part of a heavily interconnected limbic circuit, including the anterior thalamic nuclei. The underlying substrates of GRS function are undoubtedly complex.

A distinctive feature of the rodent GRS is a microcolumn-type structure found in layers I and II. In layer I, the apical dendrites of pyramidal neurons in layer II form prominent bundles (Wyss et al., 1990; Ichinohe and Rockland, 2002; Miyashita et al., 2010). After emerging from the cell body in layer II, the dendrites group together in discrete bundles as they ascend toward the pial surface. The dendritic bundles in layer $\mathrm{Ib}$ are $30-100 \mu \mathrm{m}$ in width, and the distance between bundles varies from approximately 30 $200 \mu \mathrm{m}$. Near the layer $\mathrm{Ib} / \mathrm{Ia}$ interface, the dendritic tufts diverge and spread out in layer Ia. The extension range of these tufts is thought to be confined to the distance between dendritic bundles (i.e., 30-200 $\mu \mathrm{m}$; mean, $78 \mu \mathrm{m}$, Wyss et al., 1990; Ichinohe and Rockland, 2002), and to co-localize with patches of thalamic terminations from the anteroventral (AV) thalamic nucleus (Wyss et al., 1990; Van Groen and Wyss, 1995). To further understand this microcolumn-like structure in the GRS, one of remaining questions is whether this structure extends into other layers, such as layer III/IV. Other than layer I, previous injection study showed that AV thalamic nucleus also projects to layer III/IV in the GRS (Shibata, 1993).

In this study, we examined the morphology of branches in the GRS from the AV thalamus in single axon branch resolution in order to determine whether AV axon branches in layer III/IV are branches of axons having extensive ramification in layer I, and, if so, whether the extent of arborization in layer III/IV vertically matches with that in layer I. For this purpose, we used a small volume injection of biotinylated dextran-amine (BDA) into the $\mathrm{AV}$ thalamus and reconstructing labeled single axon branches in the GRS. Our results showed that, as previously reported, the majority 
of ramifications in layer Ia of single axons from $\mathrm{AV}$ were about confined into dendritic module compartment size (i.e., 30-200 $\mu \mathrm{m}$; mean $78 \mu \mathrm{m})$. However, in three cases reconstructed, in addition to layer Ia, axons had branches in layer III/IV, which were much wider than that in layer Ia $(148-533 \mu \mathrm{m}$ : mean, $341 \mu \mathrm{m})$, suggesting layer-specific spacing of information transmission from AV occurs from single axon branches. Thus, microcolumn-type structure in the upper layer of the GRS was not strictly continuous from layer I to layer IV.

\section{MATERIALS AND METHODS}

All procedures were approved by the Animal Research Committee, Hirosaki University. Four male Wistar rats (8-10 week old: $280-$ $340 \mathrm{~g})$ were anesthetized with sodium pentobarbital $(50 \mathrm{mg} / \mathrm{kg}$, i.p.) and placed in a stereotaxic head holder. A 10\% solution of 10,000 MW BDA (Molecular Probes, Eugene, OR, USA) in 0.01 M phosphate buffer ( $\mathrm{pH}$ 7.2) were iontophoretically injected into the AV (Figure 1). The injections were performed through a glass micropipette (tip outer diameter: $50 \mu \mathrm{m}$ ) using a positive ejecting current (5-7 $\mu \mathrm{A}, 7 \mathrm{~s}$ on/7 s off) for $20-30 \mathrm{~min}$. After a survival period of 7 days, the rats were re-anesthetized and perfused transcardially with $150 \mathrm{ml}$ of saline followed by $1000 \mathrm{ml}$ of a solution composed of $4 \%$ paraformaldehyde in $0.1 \mathrm{M}$ phosphate buffer ( $\mathrm{PB}$; $\mathrm{pH}$ 7.4). The brains were removed, post-fixed for $1 \mathrm{~h}$ in the same fixative, and then kept in either $0.1 \mathrm{M} \mathrm{PB}$ or $30 \%$ sucrose in $0.1 \mathrm{M}$ PB. The following day, the brain was cut into $50-\mu \mathrm{m}$ thick serial sections in the frontal plane with a microslicer (Dosaka EM, Kyoto, Japan) or a freezing microtome (ROM 380; Yamato Koki, Asaka, Japan). The sections containing the GRS and thalamus were collected and washed in $0.1 \mathrm{M}$ phosphate-buffered saline (PBS). To visualize injected and transported BDA, the sections were incubated in PBS containing avidin-biotin-peroxidase complex (1:100 dilution; Vector, Burlingame, CA, USA) and 0.5\% Triton X-100 for $4 \mathrm{~h}$ at room temperature. The sections were again washed in PBS and then incubated in 0.1 M PBS containing 0.025\% 3,3' -diaminobenzidine tetrahydrochloride (Sigma, St. Louis, MO, USA), $0.03 \%$ nickel ammonium sulfate, and $0.008 \%$ $\mathrm{H}_{2} \mathrm{O}_{2}$. The sections were thoroughly washed in PBS, and mounted onto ABS-coated glass slides before being covered with a coverslip. The BDA-labeled axons were traced using a microscope $(\mathrm{BH} 2$; Olympus, Tokyo, Japan) equipped with a drawing tube. Sections were scanned with a $40 \times$ objective to identify labeled processes in the layer Ia. Because even small BDA injection into AV resulted in heavily dense and complexly convergent layer Ia patchy labeling, it was difficult to find such a field in that we could isolate and reconstruct a single axon within layer Ia. Candidate axons were then first reconstructed through sequential sections, by aid of a camera lucida microscope attachment, at layer Ia, and after we confirmed that candidate axons were reconstructable completely and had extensive branching at layer Ia, we followed down to other layer to white matter (WM), where axon density is low, and axons were more easily reconstructable. When layer Ia branching was not extensive, we judged that BDA filing was not enough, we did not included this study. Each branch was reconstructed using 3-10 sections, depending on tangential extensions of branches reconstructed. Both intermediate $(200 \times)$ and higher magnifications $(400 \times$ or $1,000 \times)$ were used selectively, and data images on paper

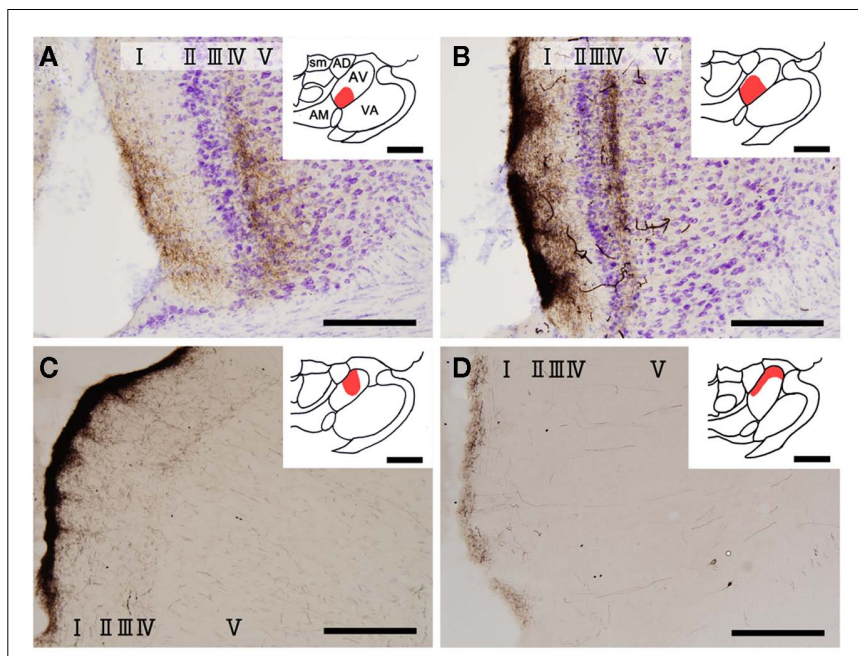

FIGURE 1 | Biotinylated dextran-amine injection sites (insets) and examples of labeled axons in the GRS. Schematic drawing of BDA injection sites in the AV thalamus (in red in insets). (A-D) Correspond to rat 1 , rat 2, rat 3, and rat 4, respectively. Tissues were counterstained for Nissl in (A,B). Abbreviations: I, layer I; II, layer II; III, layer III; IV, layer IV; V, layer V; $\mathrm{VI}$, layer $\mathrm{VI} ; \mathrm{AD}$, anterodorsal thalamic nucleus; $\mathrm{AM}$, anteromedial thalamic nucleus; $A V$, anteroventral thalamic nucleus; LD, laterodorsal thalamic nucleus; MD, mediodorsal thalamic nucleus; Rt, reticular thalamic nucleus; $\mathrm{sm}$, stria medullaris of the thalamus. Scale bar, $200 \mu \mathrm{m}, 1 \mathrm{~mm}$ (insets).

were adjusted and merged manually by xerox or by Adobe Photoshop, after scanning and digitizing. However, because of difficulty of isolation and reconstruction of an single axon at layer Ia, even 50 or more sections from each animals were scanned, number of successful reconstruction were limited (total 10 main branches), and these reconstructed axons were found in the fringe of BDA projection focus. However, four branches had sub-branches (Table 1). Thus, overall number of branches including sub-branches was 15 (Table 1), and extensions and number of boutons were measured on each sub-branch basis if main branch had sub-branches, measurements of axonal arborizations was not corrected for shrinkage. To analyze extension of branches in layer Ia and III/IV statistically, average of dorsoventral and rostrocaudal extensions of each branch were used. This number was used also for describing range of extensions of branch. Statistical analysis was done on extension and number of bounts using student- $t$ test. The level of significance was set at $P<0.05$ in all cases. After axon reconstruction was completed, to identify layer, coverslip was removed by immersing xylene for 2 days, and specimen was stained for Nissl using thionin.

\section{RESULTS}

Insets in Figure $\mathbf{1}$ show four injection sites confined to the AV thalamus. Ventral and medial injections (rats 1 and 2: insets in Figures 1A,B) resulted in labeled axons in the rostroventral GRS; the axon terminations tended to be at equal densities in layers Ia and III/IV (Figures 1A,B). The fringe of the AV projection in the GRS (but not the core) sometimes had terminations only in layer III/IV. In contrast, dorsolateral injections (rats 3 and 4: Insets in Figures 1C,D) showed less extensive axon coverage in layer III/IV (Figures 1C,D) compared with layer Ia, and the projection zone 
Table 1 | Extension of reconstructed branches in layers la and III/IV.

\begin{tabular}{|c|c|c|c|c|c|c|c|c|c|c|c|c|c|c|c|c|}
\hline & \multirow{2}{*}{$\begin{array}{l}\text { Type of branch } \\
\text { Branch No. }\end{array}$} & \multicolumn{10}{|c|}{ Type 1} & \multicolumn{5}{|c|}{ Type 2} \\
\hline & & \#1 & \#2 & \#3 & \#4 & \#5 & \#6 & & & \#7 & & \#8 & \#9 & & $\# 10$ & \\
\hline & Rat No. & 1 & 1 & 2 & 3 & 4 & 3 & & & 4 & & 1 & 2 & & 2 & \\
\hline \multirow[t]{4}{*}{ Layer la } & Dorsoventral extension ( $\mu \mathrm{m})$ & 104 & 117 & 113 & 115 & 60 & & & & & & 104 & & & & \\
\hline & Sub-branch No. & & & & & & \#6-1 & \#6-2 & \#6-3 & \#7-1 & \#7-2 & & \#9-1 & \#9-2 & \#10-1 & \#10-2 \\
\hline & Dorsoventral extension ( $\mu \mathrm{m})$ & & & & & & 138 & 89 & 161 & 89 & 182 & & 99 & 102 & 36 & 40 \\
\hline & Rostrocaudal extension ( $\mu \mathrm{m})$ & 150 & 150 & 100 & 100 & 50 & 100 & 150 & 100 & 150 & 150 & 100 & 125 & 100 & 50 & 100 \\
\hline \multirow[t]{2}{*}{ Layer III/IV } & Dorsoventral extension ( $\mu \mathrm{m})$ & 0 & 0 & 0 & 0 & 0 & 0 & 0 & 0 & 0 & 0 & 338 & 348 & 491 & 120 & 120 \\
\hline & Rostrocaudal extension ( $\mu \mathrm{m})$ & 0 & 0 & 0 & 0 & 0 & 0 & 0 & 0 & 0 & 0 & 350 & 700 & 575 & 200 & 175 \\
\hline
\end{tabular}

in the GRS was slightly more dorsal and caudal than was the case with the ventromedial injections.

Ten axon branches from the AV were reconstructed from layer I to the WM in the GRS. At the WM, the axons turned sharply in a caudal direction and entered intricate axon bundles, where they became difficult to follow and were not reconstructed further. It is worth noting that these axons that were not reconstructed may have multiple branches entering the GRS. Thus, the axons reconstructed in this study are described here as branches.

We found at least two different types of thalamocortical axon branches in the AV. Type 1 ( 7 of 10 branches) had extensive axon arborization only in layer Ia (Figure 2; Table 1), were found in all four animals (Table 1). Five of the seven type 1 branches had no collateral from the WM to layer I (Figures 2A,B), while 1 branch (branch 6) had 3 sub-branches in layer VI, and each sub-branch, interestingly, formed a highly overlapping ramification in layer Ia (Figures 2C). Another type 1 branch (branch 7) had 2 subbranches in layer VI, and both sub-branches formed an extensive ramification in layer Ia, but the tangential distance between these 2 ramifications was about $500 \mu \mathrm{m}$ (Figure 2D). The width of the ramifications of the type 1 branches in layer Ia ranged from 55$166 \mu \mathrm{m}$ (mean \pm SD: $118 \pm 23$; Table 1$)$. The number of boutons in these layer Ia ramifications ranged from 46 to 127 (mean \pm SD: $74 \pm 29$; Table 2).

Type 2 branches also ( 3 of 10 reconstructed branches) had compact branching in layer I and also showed extensive arborization in layer III/IV (Figure 3A, branch 9 and Figure 3B, branch 8). Rats 1 and 2 , which had the axon terminations tended to be at equal densities in layers Ia and III/IV, had type 2 branches. Branches 9 and 10 had 2 sub-branches. Branch 9 bifurcate at layer VI (Figure 3A), and branch 10 at layer $\mathrm{V}$ (not shown). Of branch 9, one sub-branch had extensive arborization in both layer Ia and layer III/IV, and the other sub-branch had only meager ramification in layer Ia, but extensive ramification at layer III/IV (Figure 3A). Of branch 10, general characteristic is similar to those of branch 9, but branch 9 is in general smaller than branch 9 (not shown). The extension and the morphology of type 2 branches in layer Ia were similar to those of the type 1 branches $(43-112 \mu \mathrm{m}$, mean $\pm \mathrm{SD}$ : $85 \pm 26 \mu \mathrm{m}$ : Table 1). However, bouton number of type 2 in layer I (bouton count: $5-73$, mean \pm SD: $29 \pm 25$; Table 2 ) is statistically significantly smaller than ones of type 1 . The extensions of the type 2 branches in layer III/IV (range: $148-533 \mu \mathrm{m}$, mean \pm SD: $342 \pm 168 \mu \mathrm{m}$ : Table 1) were statistically more divergent than those in layer I either of type 1 only, type 2 only, mixture of type 1 and 2, and, obviously, but not statistically tested, than reported dendritic module size (i.e., 30-200 $\mu \mathrm{m}$; mean $78 \mu \mathrm{m}$; Wyss et al., 1990; Ichinohe and Rockland, 2002). Number of boutons in these layers (45-148, $92 \pm$ 45; Tables 1 and 2; Figures 3A,B) was also numerous than those of layer Ia, but not statistically significant.

\section{DISCUSSION}

The present study using extracellular labeling methods showed that the AV has a variety of projection types with extensive arborization occurring in layer Ia (type 1), layers Ia and III/IV (type 2). As previously reported (Wyss et al., 1990), both axon branch types had extensive ramifications in layer Ia, with lateral extensions within the range of each apical dendritic bundle from layer II pyramidal neurons (i.e., 30-200 $\mu \mathrm{m}$ ). Our study also showed that AV axons could branch in the deep layers, and those branches could target the same module in layer I (branch 6), or target the different modules, which separated each other in more than $500 \mu \mathrm{m}$ distance (branches 7, 9, and 10), suggesting that a single AV axon can simultaneously target distant dendritic modules in layer I/II. Whole modules targeted by a single axon should be determined by more clearly visible tracers (i.e., single virus infection methods: Kuramoto et al., 2009) or juxtacellular recording and staining (Deschênes et al., 1996).

Type 2 branches displayed extensive collateral arborization in layer III/IV statistically wider than layer Ia ramifications either of type 1 only, type 2 only, mixture of type 1 and 2, and, obviously, but not statistically tested, than reported dendritic module size (i.e., 30-200 $\mu \mathrm{m}$; mean $78 \mu \mathrm{m}$; Wyss et al., 1990; Ichinohe and Rockland, 2002). This results suggest that layers I/II and III/IV may have different spacing of information transmission of a AV thalamic axon. Thus, at least, conspicuous modules in layer I/II of GRS seems not to extend just into layer III/IV at the same spatial scale. This finding reminds us the fact that neuronal cell bodies in layer II of the GRS are only labeled by retrograde-tracer injections exactly contralateral to the labeled cells, i.e., there is a "point-to-point" organization (Sripanidkulchai and Wyss, 1987). This organization contrasts with that seen in layers III and V, where commissural projections arise from an area 10-20 times larger than the injection. This layer-specific organization is different from other areas of the sensory cortex (e.g., the barrel cortex: Aronoff and Petersen, 2008), where axonal arborization through layers tends to be confined to a single columnar organization. 


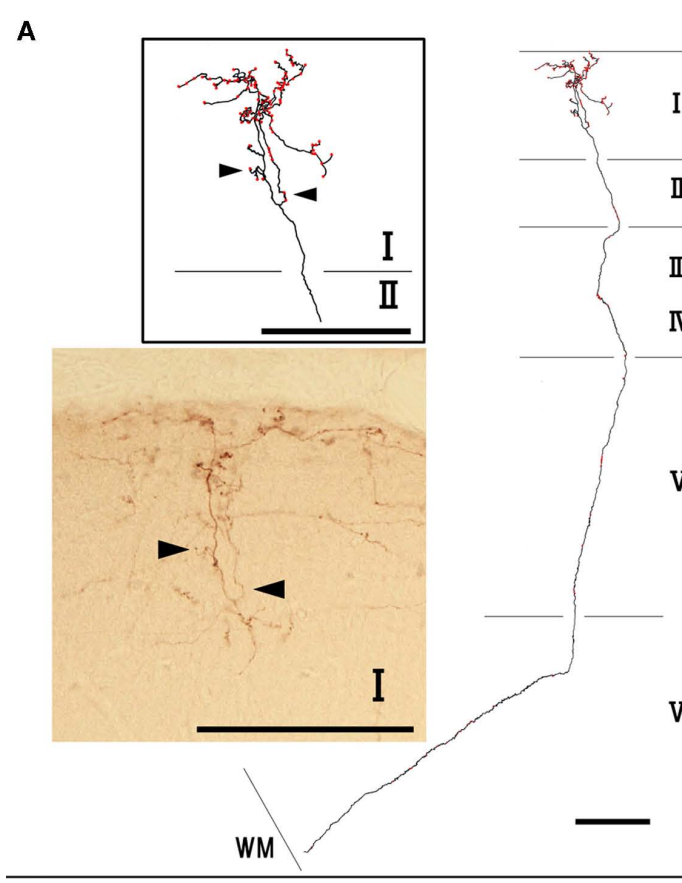

C

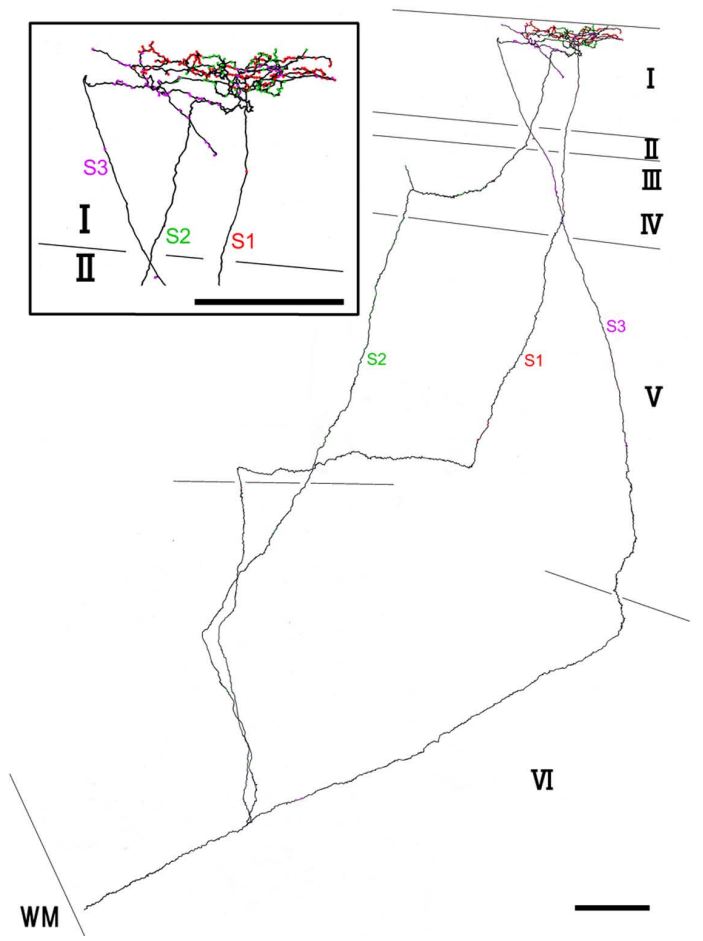

FIGURE 2 | Examples of type 1 axon branches. (A) branch 1; (B) branch 2; (C) branch 6; (D) branch 7. Red dots on axons represent boutons. Insets within rectangles are enlarged images, mainly in layer I of each branch. Photographic inset in (A) is from a single section of the branch inset
B

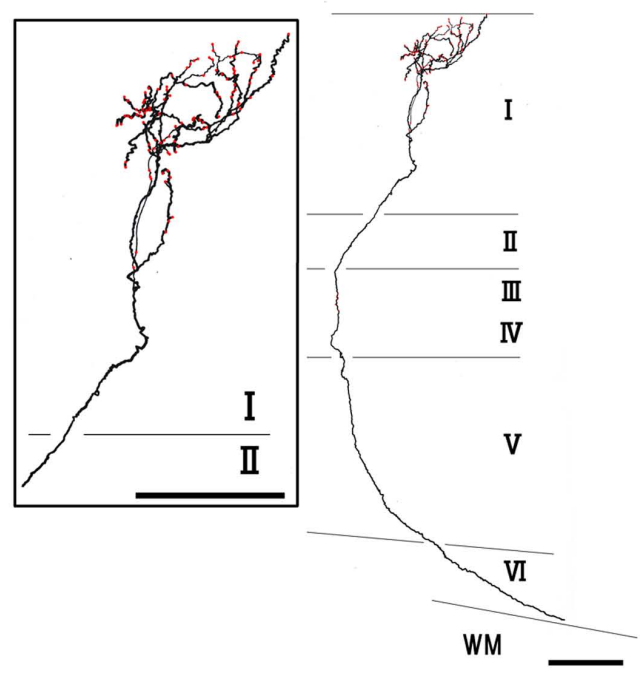

VI

WM

D
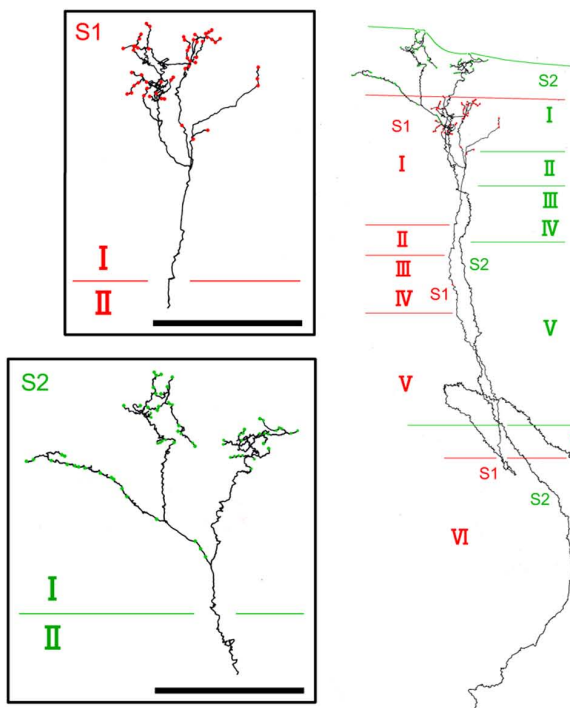

V

V

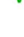

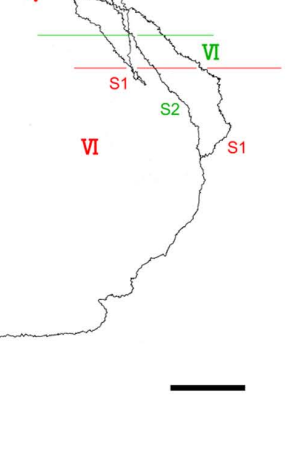

WM
Other layer-specific and/or input-origin (e.g., subiculum, Ichinohe et al., 2008) specific spacing of neuronal components in the GRS should be examined by anatomical and functional (e.g., electrophysiology and two-photon imaging) methods in the future above. Arrowheads point to the same portion of the branches. Abbreviations: I, layer I; II, layer II; III, layer III; IV, layer IV; V, layer V; VI, layer VI; S1, sub-branch 1; S2, sub-branch 2; S3, sub-branch 3. Scale bar $100 \mu \mathrm{m}$.

in view of interaction of each neuronal components. In that sense, it is interesting, now, using molecular technique, we could use animals, in which dendritic bundles can be visualized using by electroporation of reporter fluorescent protein gene and/or 
Table 2 | Number of boutons on reconstructed branches in each layer.

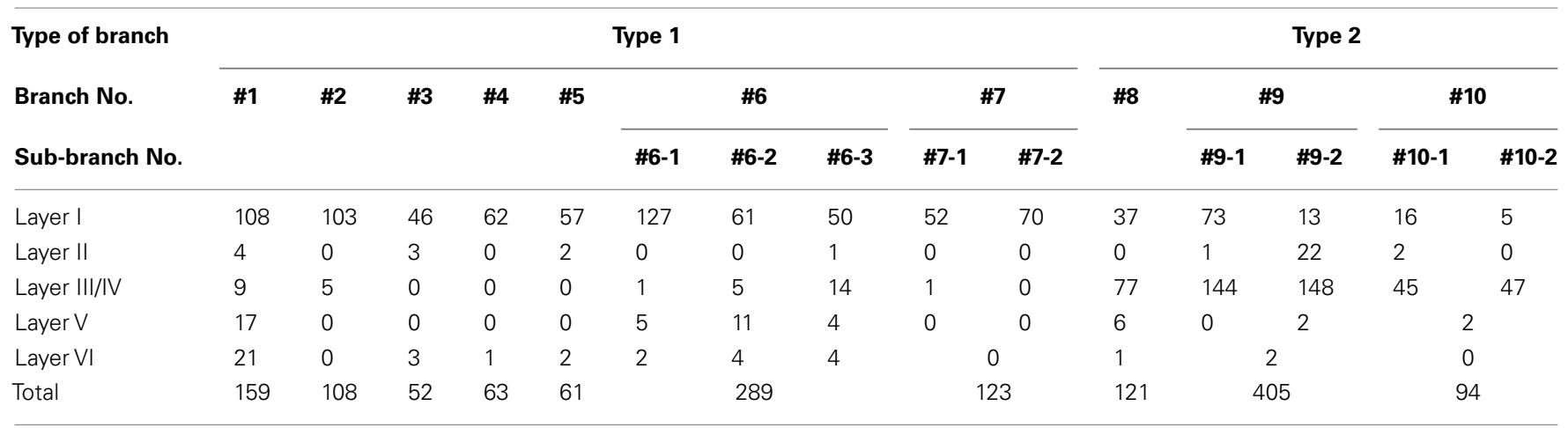

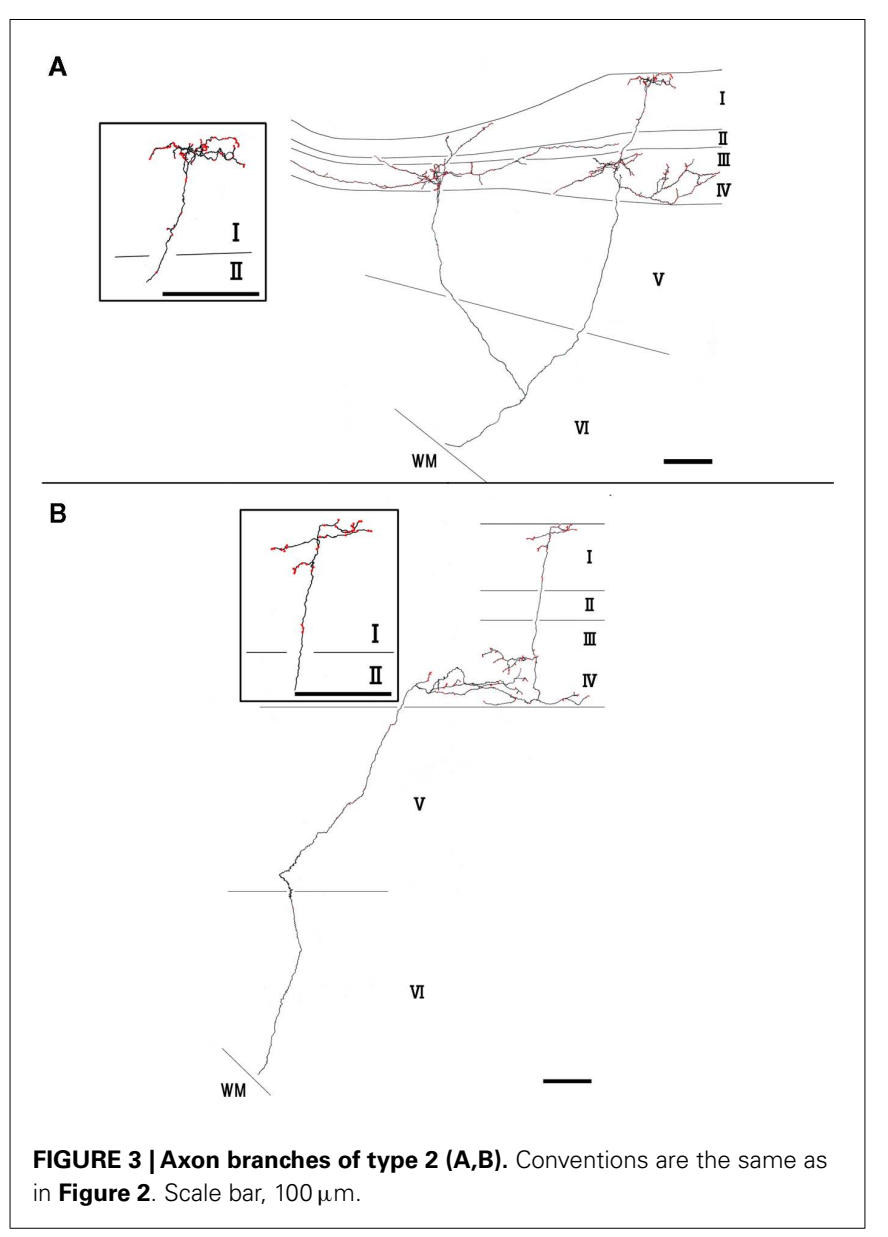

\section{REFERENCES}

Aggleton, J. P. (2010). Understanding retrosplenial amnesia: insights from animal studies. Neuropsychologia 48, 2328-2338.

Aronoff, R., and Petersen, C. C. (2008). Layer, column and cell-type specific genetic manipulation in mouse barrel cortex. Front. Neurosci. 2:1. doi:10.3389/neuro.01.001.2008

Cooper, B. G., and Mizumori, S. J. (2001). Temporary inactivation of the retrosplenial cortex causes a transient reorganization of spatial coding in the hippocampus. J. Neurosci. 21, 3986-4001.

Deschênes, M., Bourassa, J., Doan, V. D., and Parent, A. (1996). A singlecell study of the axonal projections arising from the posterior intralaminar thalamic nuclei in the rat. Eur. J. Neurosci. 8, 329-343.

Garden, D. L., Massey, P. V., Caruana, D. A., Johnson, B., Warburton, E.

viral vector in in vivo (Miyashita et al., 2010; Zgraggen et al., 2011).

The existence of type 1 and 2 branches may guide the way to solve a discrepancy related to the target layer of AV terminals. One group of studies has emphasized that layer Ia is almost the sole target of AV terminals (Van Groen and Wyss, 1995); the other group has shown AV terminals in both layers Ia and III/IV (Shibata, 1993). This discrepancy may be derived from a tracer injection falling in a segregated mass of different types of neurons within the AV. Our study showed that, although type 1 branches were found in all animals, type 2 branches were only found in rats 1 and 2, which had the labeled axon terminations at equal densities in layers Ia and III/IV. Van Groen and Wyss (1995) may have injected tracers into the AV, where type 1 neurons aggregated, but, Shibata (1993) may have injected tracers into the area, where both type 1 and 2 neurons existed. In consistent with this idea, our results showed that different position of BDA injection within $\mathrm{AV}$ resulted in either dense terminals in layer Ia with weak labeling in layer III/IV or equal density in layer Ia and III/IV (this study). Another possibility is that potential preferential uptake of the different tracer (i.e., tracer, PHA-L, Shibata, 1993; tracer, Fluoro-Ruby, Van Groen and Wyss, 1995) by type 2 and/or type 1 branches, respectively. Recent methods for more clearly visible single labeling of small number of neurons (Deschênes et al., 1996; Kuramoto et al., 2009) can give more concrete answer for the hypothesis above.

\section{ACKNOWLEDGMENTS}

This study was supported by a Grant-in-Aid for Scientific Research on Innovative Areas, "Face perception and recognition," by the Ministry of Education, Science, Sports, and Culture, Japan and Grant for Hirosaki University Institutional Research.

C., Aggleton, J. P., and Bashir, Z. I. (2009). Anterior thalamic lesions stop synaptic plasticity in retrosplenial cortex slices: expanding the pathology of diencephalic amnesia. Brain 132, 1847-1857.

Ichinohe, N., Knight, A., Ogawa, M., Ohshima, T., Mikoshiba, K., Yoshihara, Y., Terashima, T., and Rockland, K. S. (2008). Unusual patchmatrix organization in the retrosplenial cortex of the reeler mouse and
Shaking rat Kawasaki. Cereb. Cortex 18, 1125-1138.

Ichinohe, N., and Rockland, K. S. (2002). Parvalbumin positive dendrites co-localize with apical dendritic bundles in rat retrosplenial cortex. Neuroreport 13, 757-761.

Kuramoto, E., Furuta, T., Nakamura, K. S., Unzai, T., Hioki, H., Kaneko, T. C., Unsai, T., Hioki, H., and Kaneko, T. (2009). Two types of thalamocortical 
projections from the motor thalamic nuclei of the rat: a single neuron-tracing study using viral vectors. Cereb. Cortex 19, 2065-2077.

Miyashita, T., Wintzer, M, Kurotani, T., Konishi, T., Ichinohe, N., and Rockland, K. S. (2010). Neurotrophin3 is involved in the formation of apical dendritic bundles in cortical layer 2 of the rat. Cereb. Cortex 20, 229-240.

Shibata, H. (1993). Efferent projections from the anterior thalamic nuclei to the cingulate cortex in the rat. J. Comp. Neurol. 330, 533-542.

Sripanidkulchai, K., and Wyss, J. M. (1987). The laminar orga- nization of efferent neuronal cell bodies in the retrosplenial granular cortex. Brain Res. 406, 255-269.

Van Groen, T., and Wyss, J. M. (1995). Projections from the anterodorsal and anteroventral nucleus of the thalamus to the limbic cortex in the rat. J. Comp. Neurol. 358, 584-604.

Vann, S. D., Aggleton, J. P., and Maguire, E. A. (2009). What does the retrosplenial cortex do? Nat. Rev. Neurosci. 10, 792-802.

Wyss, J. M., Van Groen, T., and Sripanidkulchai, K. (1990). Dendritic bundling in layer I of granular retrosplenial cortex: intracellular labeling and selectivity of innervation. J. Comp. Neurol. 295, 33-42.

Zgraggen, E, Boitard, M., Roman, I. Kanemitsu, M., Potter, G., Salmon, P., Vutskits, L., Dayer, A. G., and Kiss, J. Z. (2011). Early postnatal migration and development of layer ii pyramidal neurons in the rodent cingulate/retrosplenial cortex. Cereb. Cortex [Epub ahead of print].

Conflict of Interest Statement: The authors declare that the research was conducted in the absence of any commercial or financial relationships that could be construed as a potential conflict of interest.
Received: 04 August 2011; accepted: 23 September 2011; published online: 11 October 2011.

Citation: Odagiri S, Meguro R, Asano $Y$, Tani $T$ and Ichinohe $N$ (2011) Single axon branching analysis in rat thalamocortical projection from the anteroventral thalamus to the granular retrosplenial cortex. Front. Neuroanat. 5:63. doi: 10.3389/fnana.2011.00063 Copyright (C) 2011 Odagiri, Meguro, Asano, Tani and Ichinohe. This is an open-access article subject to a nonexclusive license between the authors and Frontiers Media SA, which permits use, distribution and reproduction in other forums, provided the original authors and source are credited and other Frontiers conditions are complied with. 